\title{
Effectiveness of High-Intensity Interval Exercise on Serum Dopamine Level and Improvement of Perceptual-Motor Skills in Male Students with Hyperactivity/Attention Deficit Disorder
}

\section{ART ICLE INFO}

\section{Article Type}

Original Research

\section{Authors}

Torabi F* $P h D$

Ebrahim R. ${ }^{1} M S c$,

Hemayattalab R. ${ }^{2} P h D$,

Ramezankhani A. ${ }^{3} P h D$

How to cite this article
Torabi F, Ebrahim R, Hemayattalab
R, Ramezankhani A. Effectiveness
of High-Intensity Interval Exercise
on Serum Dopamine Level and
Improvement of Perceptual-Motor
Skills in Male Students with
Hyperactivity/Attention Deficit
Disorder. Horizon of Medical
Sciences. 2017;23(1):35-39.

*Physical Education Behavior Department, Tehran-Jonoob Center, Payam-e-Noor University, Tehran, Iran

${ }^{1}$ Physical Education Behavior Department, Tehran-Jonoob Center, Payam-e-Noor University, Tehran, Iran

${ }^{2}$ Motor Behavior Department, Physical Education \& Sport Sciences Faculty, Tehran University, Tehran, Iran ${ }^{3}$ Exercise Physiology Department, Physical Education \& Sport Sciences Faculty, Tehran University, Tehran, Iran

\section{Correspondence}

Address: Payam-e-Noor University, Tehran-Jonoob Branch, Nejatollahi Street, Karim Khan Avenue, Tehran, Iran

Phone: +98 (21) 88807617

Fax: +98 (21) 88807617

torabift@yahoo.com

\section{Article History}

Received: March 2,2016

Accepted: August 27, 2016

ePublished: January 19, 2017

\section{A B S T RAC T}

Aims Known by hyperactivity, inattentiveness, and impulsiveness, the attention deficit hyperactivity disorder (ADHD) is considered as a behavioral disorder in the children, as well as in the adolescents. The disorder might also damage their motor skill procedure. The aim of the study was to determine the effectiveness of 6-week high intensity interval exercise on the serum dopamine levels and the improvement of perceptual-motor performance in boys with ADHD. Materials \& Methods In the controlled pretest-posttest semi-experimental study, 20 adolescent male students with ADHD of the eastern Tehran schools were studied in 2015. The subjects, selected by random sampling method, were randomly divided into two groups including experimental $(n=10)$ and control $(n=10)$ groups. 6-week high intensity interval training (3 days a week) was conducted in experimental group. The anthropometric indices, dopamine levels, and perceptual-motor performance scores were measured both at the beginning and at the end of the course. Data was analyzed by SPSS 16 software using paired T and independent T tests. Findings In the experimental group, the dopamine levels significantly increased at the posttest stage compared to the pretest $(\mathrm{p}=0.01)$, while BMI $(\mathrm{p}=0.001)$ and body fat percentage $(p=0.002)$ significantly decreased. In addition, the motor skill score significantly increased in experimental group $(\mathrm{p}=0.001)$. No variable was significantly changed in control group during the 6 weeks $(\mathrm{p}>0.05)$.

Conclusion 6-week high intensity interval exercise improves perceptual-motor performance and increases serum dopamine levels in boys with ADHD.

\section{Keywords High Intensity Interval Training; Dopamine; Attention Deficit Hyperactivity} Disorder ; Motor Skills

\section{I T A T I O N L I N KS}

[1] Theoretical explanation of the disorder, attention deficit/hyperactivity over pattern of behavioral ... [2] Attention-deficit hyperactivity ... [3] Combining parent and child training for young children with ... [4] Physical activity experiences of boys with and without ... [5] Effects of physical activity intervention on motor proficiency and physical fitness in children with ADHD: An exploratory ... [6] Development of attentional processes in ADHD and normal ... [7] Effect of stimulant medication use by children with adhd on heart rate and perceived ... [8] Short term cardiovascular effects of methylphenidate and ... [9] Influence of the aerobic exercise plan and group theraplay on the balance of children with attention deficit and ... [10] Swimming exercise alleviates the symptoms of attention-deficit hyperactivity disorder in spontaneous hypertensive ... [11] Annual Research Review: Infant development, autism, and ADHD--early pathways to emerging ... [12] Inhibition and the right inferior frontal cortex: One decade ... [13] Comorbidity of ADHD and anxiety: From basic to applied ... [14] Motor function in school-aged children with attention-deficit/ hyperactivity disorder in ... [15] A Physical Activity Program Improves Behavior and Cognitive Functions in Children with ADHD: An Exploratory ... [16] Acute exercise effects on measures of attention and impulsivity in children with attention deficit/hyperactivity ... [17] The effects of massage therapy \& exercise therapy on children/adolescents with attention deficit hyperactivity ... [18] The effect of physical exercise on the development of gross motor skills in children with attention deficit / hyperactivity ... [19] Motor development of children with attention deficit hyperactivity ... [20] Eating pathology among adolescent girls with attention-deficit/hyperactivity ... [21] Prevalence of obesity in ... [22] Obesity and ADHD may represent different ... [23] Investigation of the effect of ... [24] Attention-deficit/hyperactivity ... [25] Group aquatic aerobic exercise for ... 


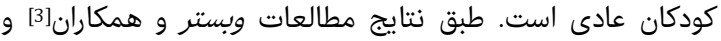

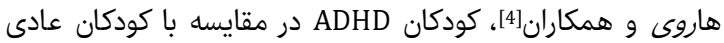

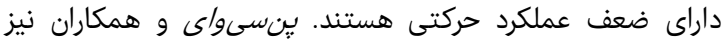

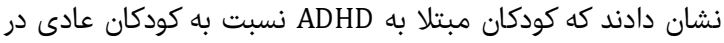

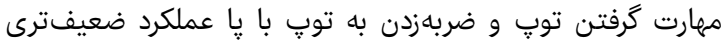

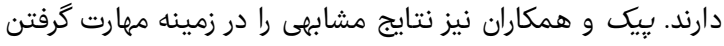

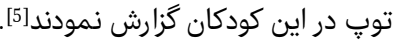

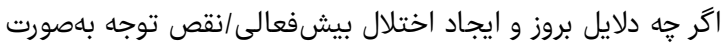

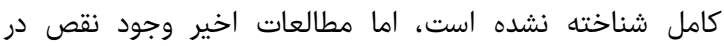

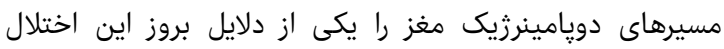

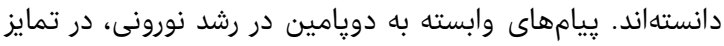

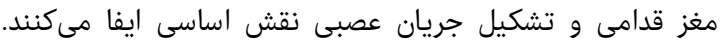

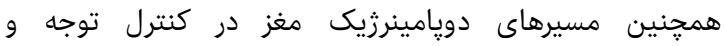
تكانشخرى نيز دركير هستند ف6].

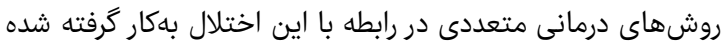

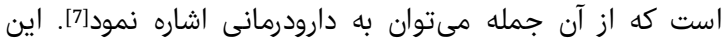

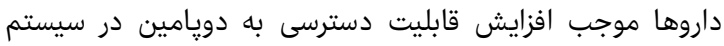

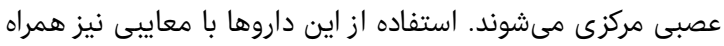

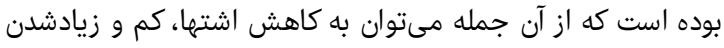

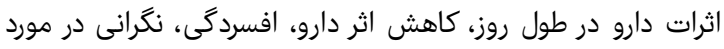

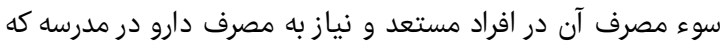

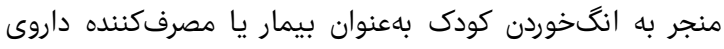

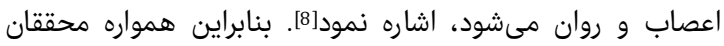

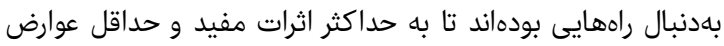

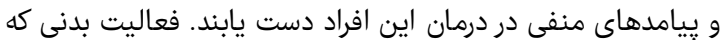

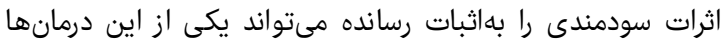

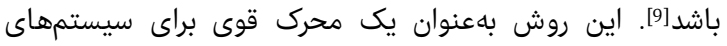

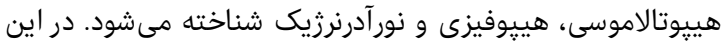

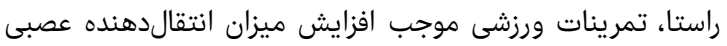

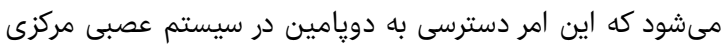

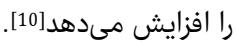

نظرات موافق بسيارى در ارتباط با يِيشرفت عملكرد اجرايى از طريق برديق

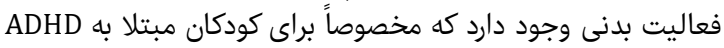

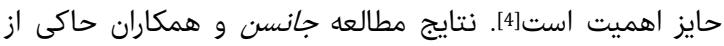

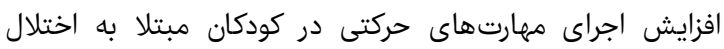

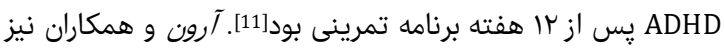

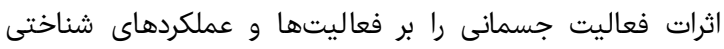

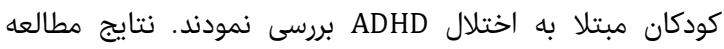

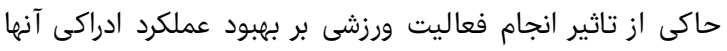

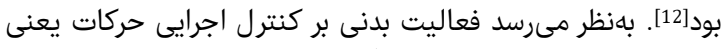

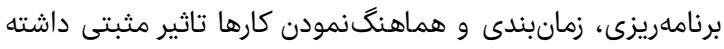

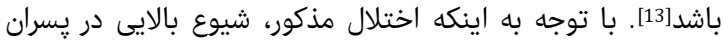

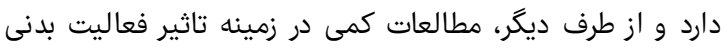

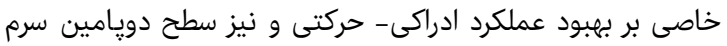

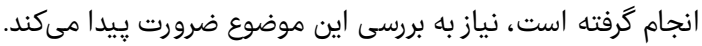

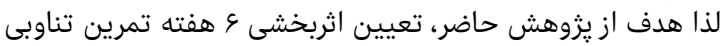

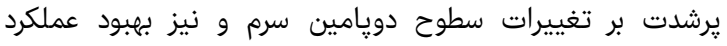

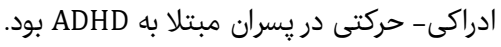

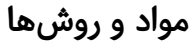

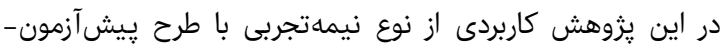

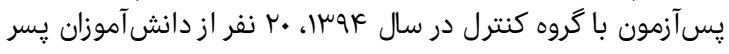

دوره سץ، شماره ا، زمستان وهـ|
اثربخشى تمرين تناوبى يرشدت بر ميزان دوران دوريامين

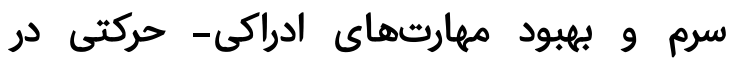

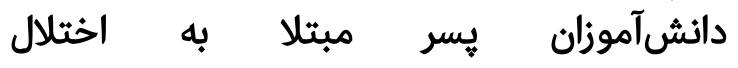
بيشفعالى/نقص توجه

\author{
"فرناز ترابى" \\ كروه تربيت بدنى، مركز تهران جنوب، دانيق دانشكاه ييام نور، تهران، ايران

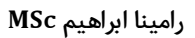
كروه تربيت بدنى، مركز تهران جنوب، دانشكاه يِيام نور، تهران، ايران

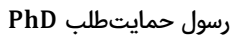
كروه رفتار حركتى، دانشكده تربيت بدنى و علوم ورزشى، دانشكاه تهران، تهران، ايران

اعظم رمضان إنانى PhD كروه فيزيولوزى ورزش، دانى دانشكده تربيت بدنى و علوم ورزشى، دانشكاه تهران، تهران، ايران

جكيده اهداف: اختلال بيشفانهالى انقص توجه، يك اختلال رفتارى در كودكان و

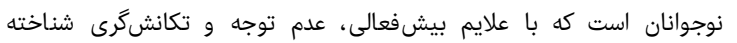

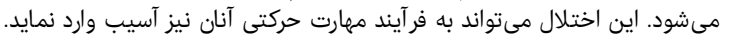

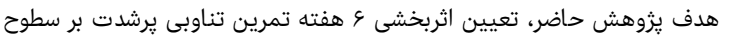

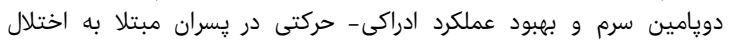

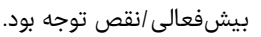

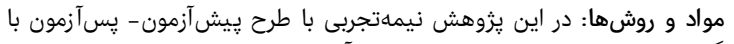

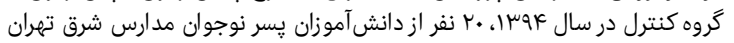

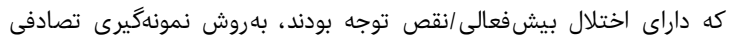

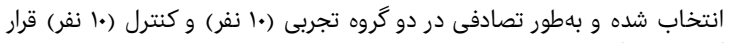

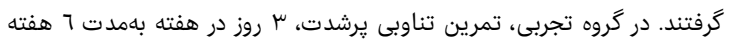

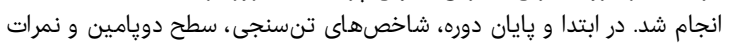

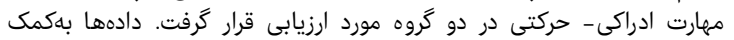

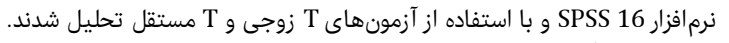

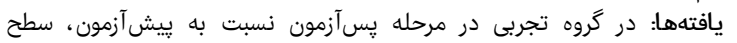

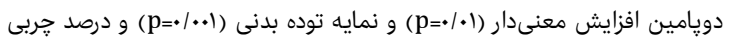

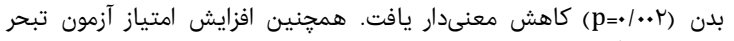

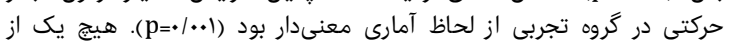

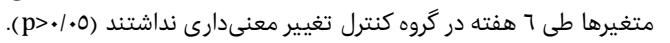

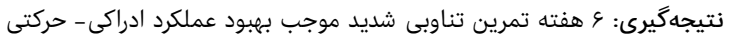

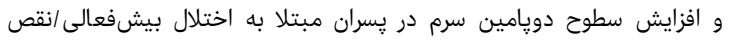

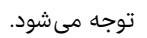
كليدوازهها: تمرين تناوبى يرشدت، دويامين، اختلال بيشفعالى/نقص توجه، مهارت حركتى

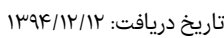

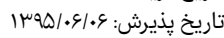

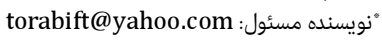

\section{مقدمه}

اختلال بيشفعالى/نقص توجه (ADHD)، يك نقص در سيستم

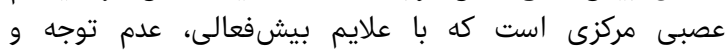

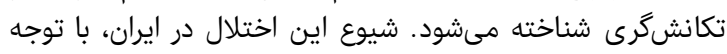

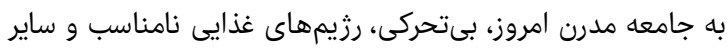

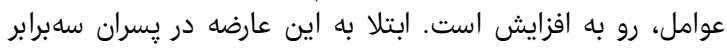

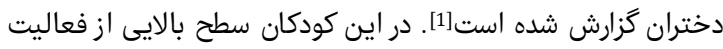

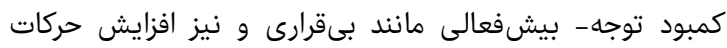

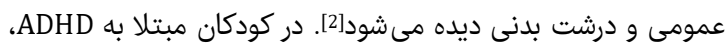

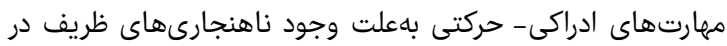

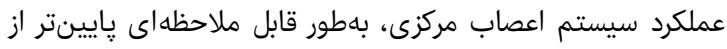

فصل نامه علمى - يخروهشى افق دانش 


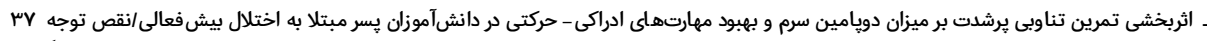

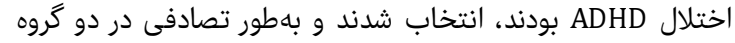

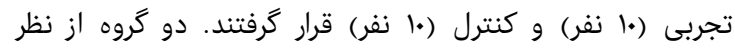

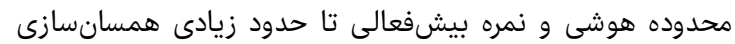

شدند.

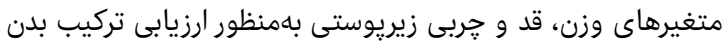

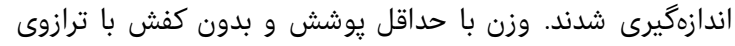

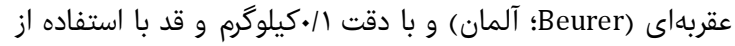

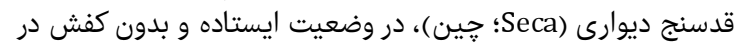

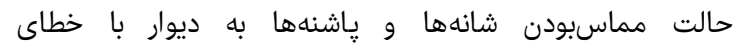

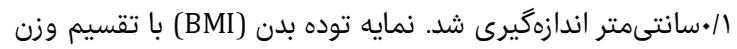

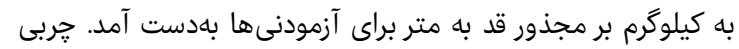

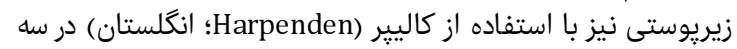

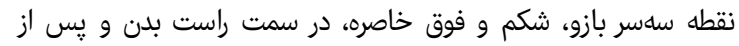

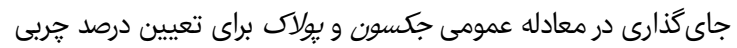
محاسبه شد.

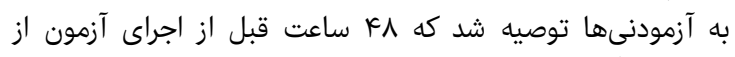

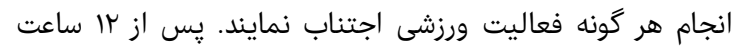

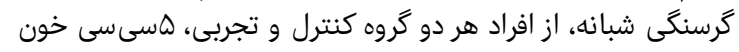

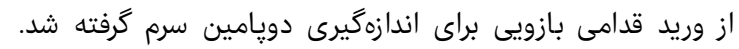

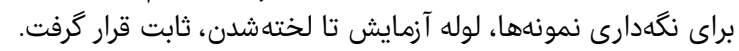

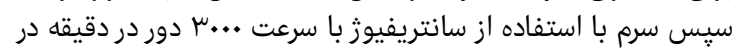

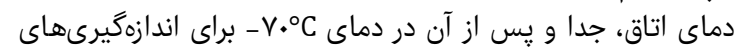

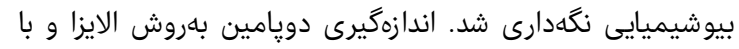

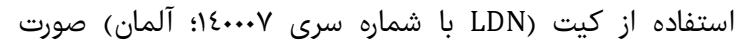

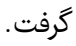

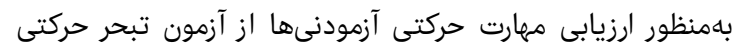

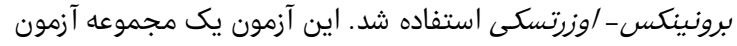

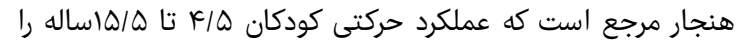

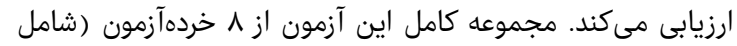

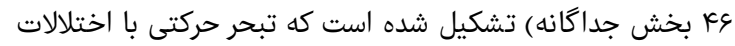

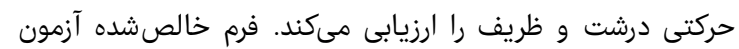

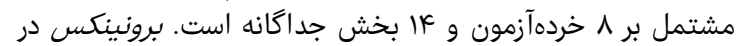

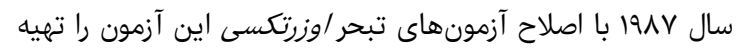

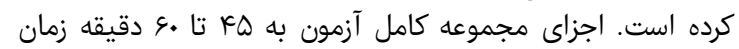

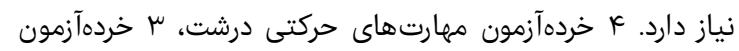

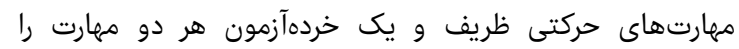

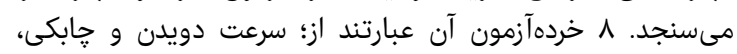

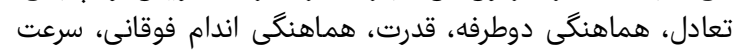

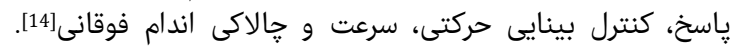

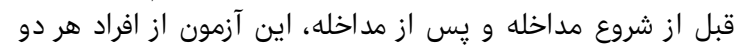

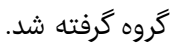

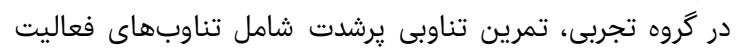

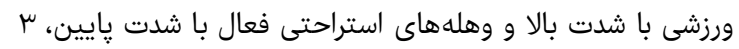

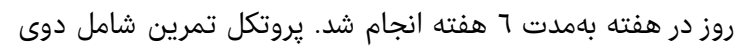
•r.r.

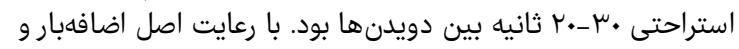

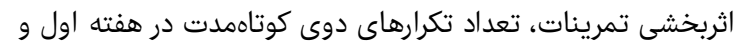

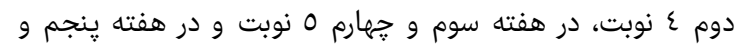

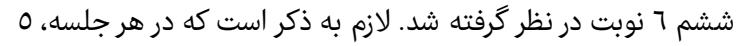

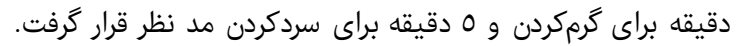

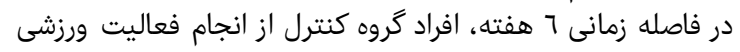
منع شدند.

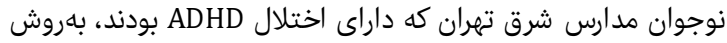

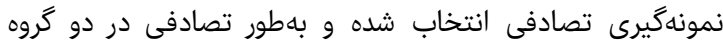

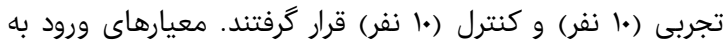

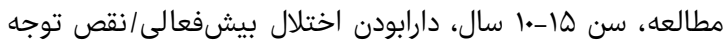

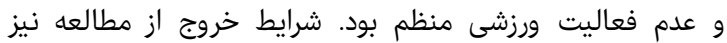

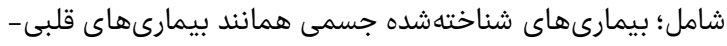

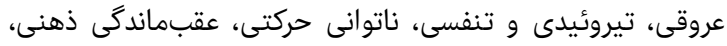

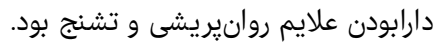

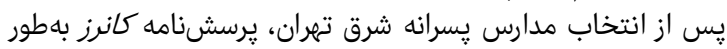

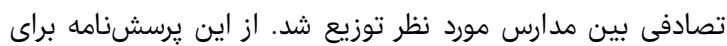

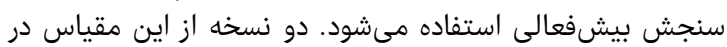

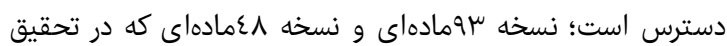

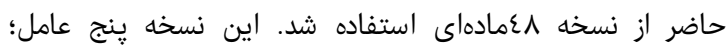

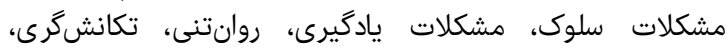

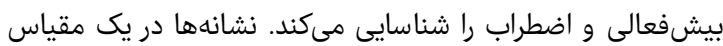

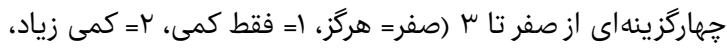

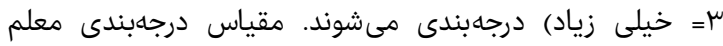

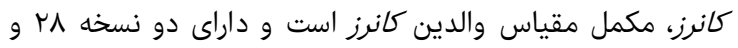

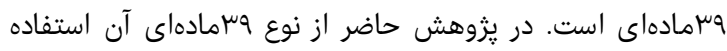

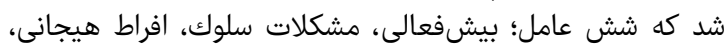

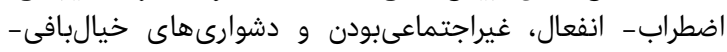

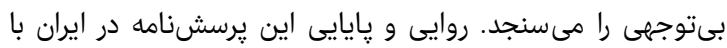

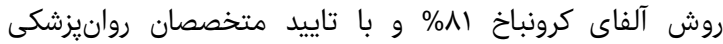
مناسب ارزيابى شد [14].

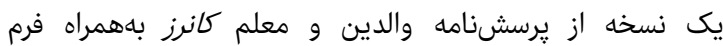

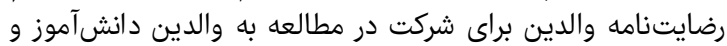

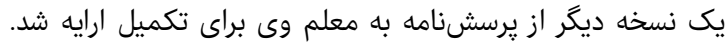

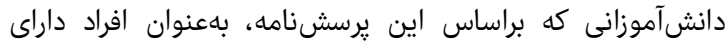

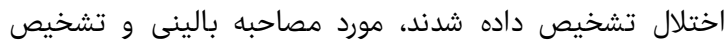

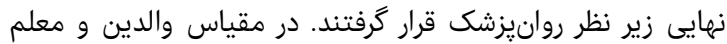

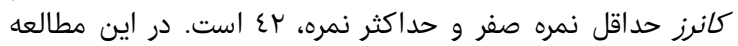

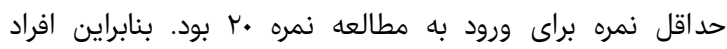

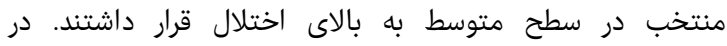

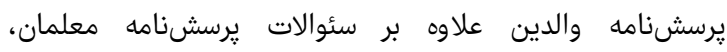

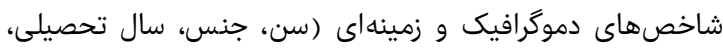

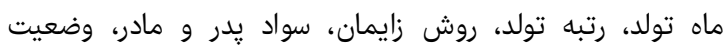

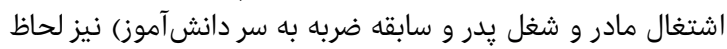

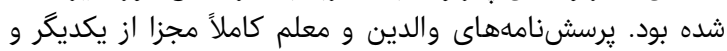

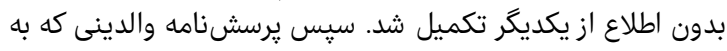

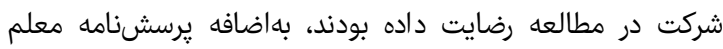

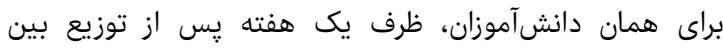

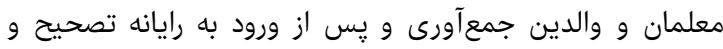

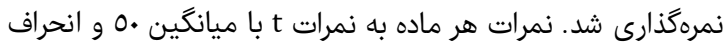

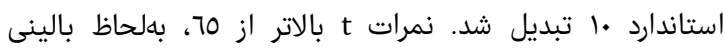

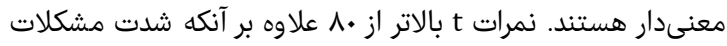

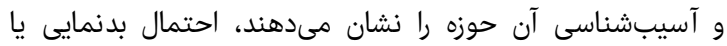

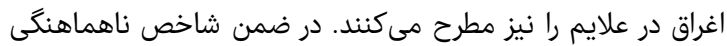

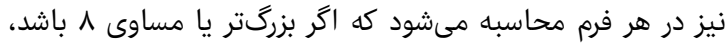

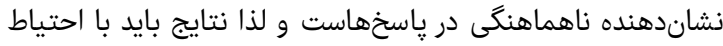

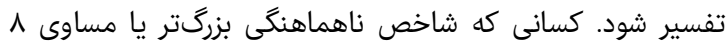

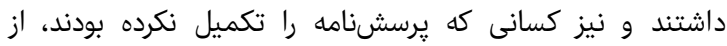

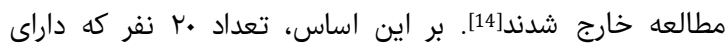


نوجوانان مبتلا به اختلال بيشفعالى/نقص توجه مىشود. نتايج

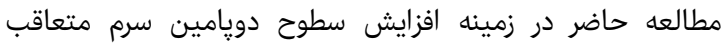

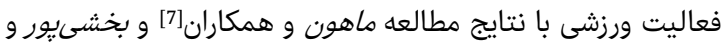

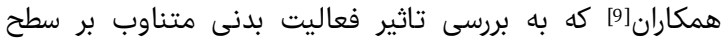

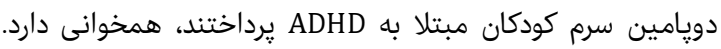

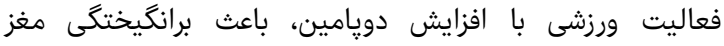

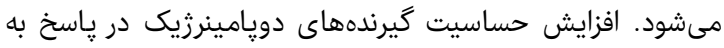

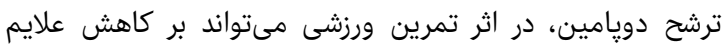

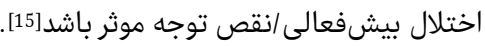

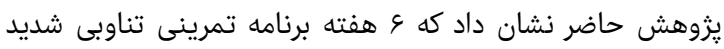

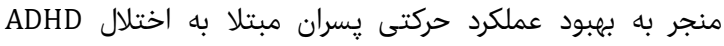

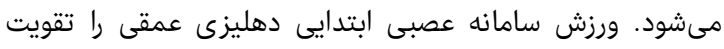

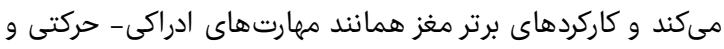

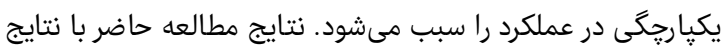

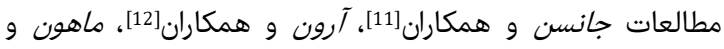

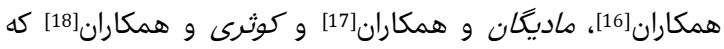

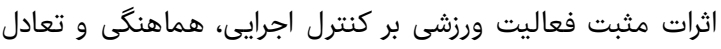

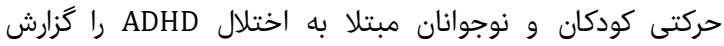

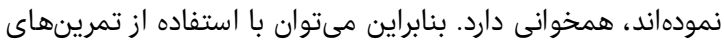

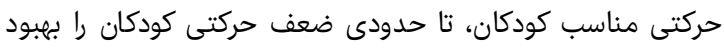

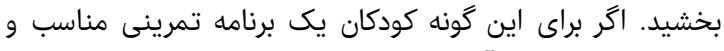

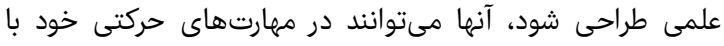
موفقيت عمل كنند [19].

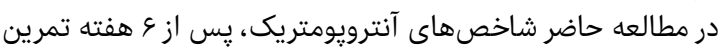

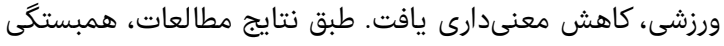

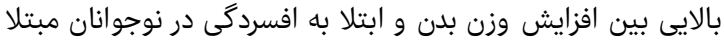

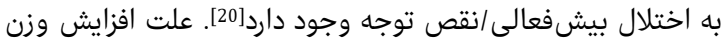

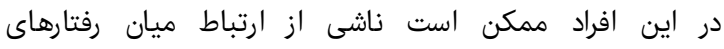

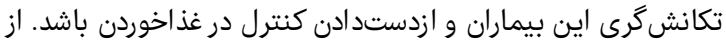

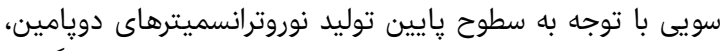

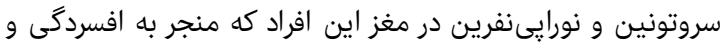

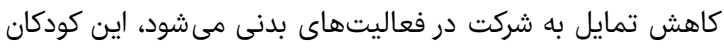

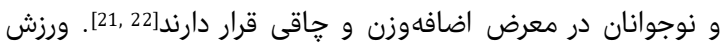

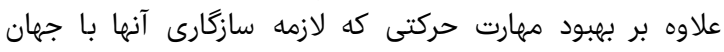

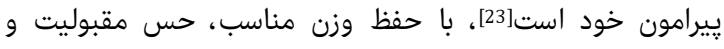

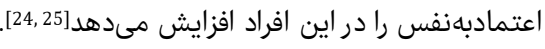

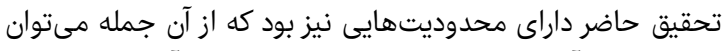

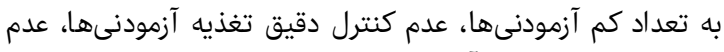

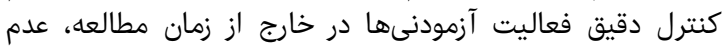
كنترل وضعيت روحى و روانى آنها و انتخاب حجم آندار نمونه اشاره

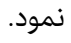
از آنجا كه اين كودكان با داشتن سطح آمادگى جسمانى يايين دجار

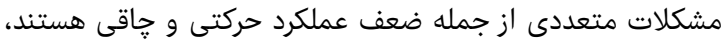

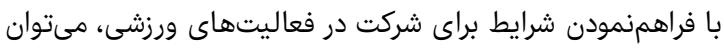

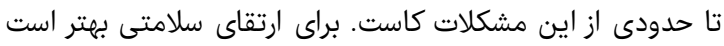

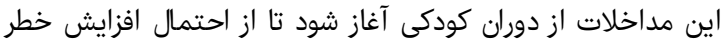

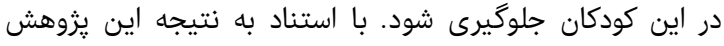

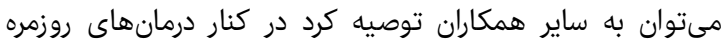

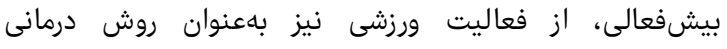

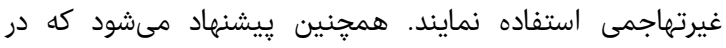
مطالعات آتى، ورزشهاى رسمى و هدفمند نيز مورد استفاده قرار

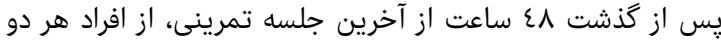

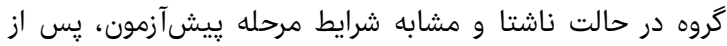

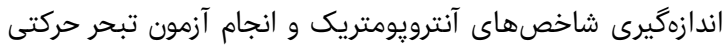

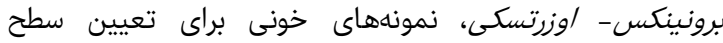

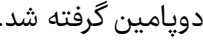

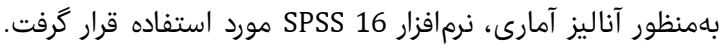

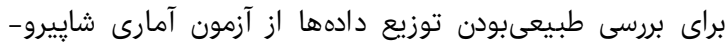

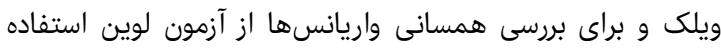

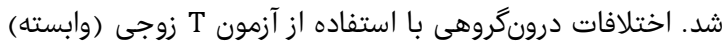

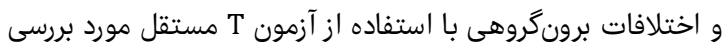

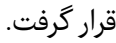

\section{يافتهها}

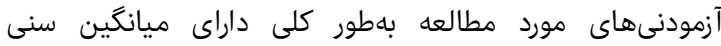

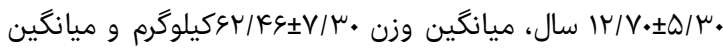

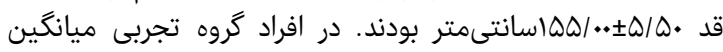

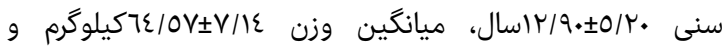

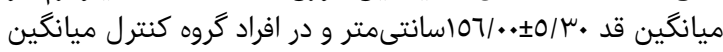

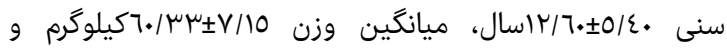

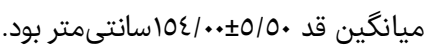

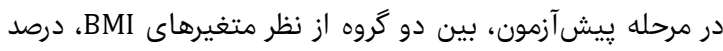

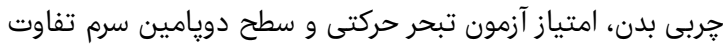

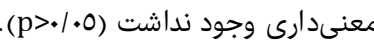

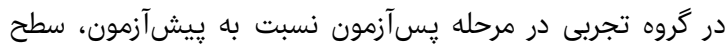

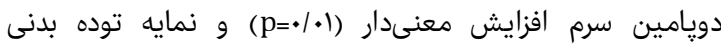
(p=•/.1)

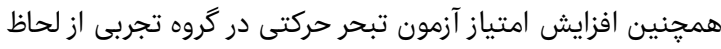

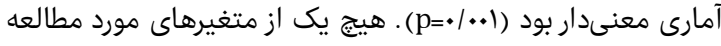

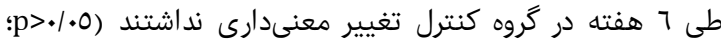

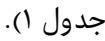

\begin{tabular}{|c|c|c|}
\hline 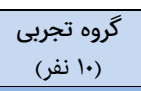 & $\begin{array}{c}\text { ك.روه كنترل } \\
\text { (. نفر) }\end{array}$ & شاخص \\
\hline $\begin{array}{l}r \varepsilon / \varepsilon \mu_{ \pm} \mu^{\prime} / O r \\
r / O \Lambda \pm \mu^{\prime} / A r\end{array}$ & $\begin{array}{l}r 7 / 97 \pm r / 70 \\
r V / .\urcorner \pm / / 9 Y\end{array}$ & يَيشآيه توده بدنى (كيلوكرم بر مترمربع) \\
\hline & & ميزان خקربى بدن (درصد) \\
\hline 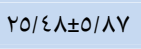 & $r V / \varepsilon O \pm \varepsilon / \cdot \Lambda$ & ي ييشآزمون \\
\hline$r r / 7 \mu_{ \pm 0 / 01}$ & או & يِسآزمون \\
\hline & & سطح دويامين سرم (نانوكرم در ليتر) \\
\hline $1 / 47 \pm \cdot 107$ & $1 / \cdot \wedge \pm \cdot / 7 \wedge$ & ييشآزمون \\
\hline $1 / 77 \pm . / 71$ & $. / 9 Y_{ \pm} \cdot / \mathrm{VO}$ & يسآزمون \\
\hline $\begin{array}{l}\mu 0 / 70 \pm 7 / \mu q \\
\varepsilon r / .7 \pm q / \mu .\end{array}$ & $\begin{array}{l}\mu \varepsilon / / V \pm \mu / 70 \\
\mu V / \mid V \pm \varepsilon / 7 V\end{array}$ & 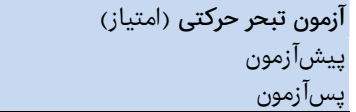 \\
\hline
\end{tabular}

بحث

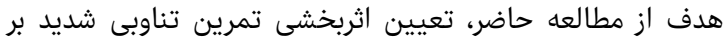

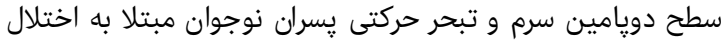

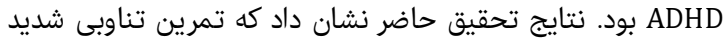

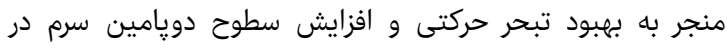




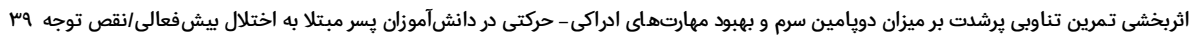

Research Review: Infant development, autism, and ADHD--early pathways to emerging disorders. J Child Psychol Psychiatry. 2015;56(3):228-47.

12- Aron AR, Robbins TW, Poldrack RA. Inhibition and the right inferior frontal cortex: One decade on. Trends Cogn Sci. 2014;18(4):177-85.

13- Jarrett MA. Comorbidity of ADHD and anxiety: From basic to applied research. ADHD Rep. 2013;21(5):1-6.

14- Cho H, Ji S, Chung S, Kim M, Joung Y. Motor function in school-aged children with attentiondeficit/hyperactivity disorder in Korea. Psychiatr Invest. 2014;11(3):223-7.

15- Verret C, Guay MC, Berthiaume C, Gardiner P, Béliveau L. A Physical Activity Program Improves Behavior and Cognitive Functions in Children with ADHD: An Exploratory Study. J Atten Disord. 2012;16(1):71-80.

16- Mahon AD, Dean RS, McIntosh DE, Marjerrison AD, Cole AS, Woodruff ME. Acute exercise effects on measures of attention and impulsivity in children with attention deficit/hyperactivity disorder. J Educ Dev Psychol. 2013;3(2):65-73.

17- Maddigan B, Hodgson P, Heath S, Dick B, St John K, McWilliam. The effects of massage therapy \& exercise therapy on children/adolescents with attention deficit hyperactivity disorder. Can Child Adolesc Psychiatr Rev. 2003;12(2):40-3.

18- Kosari S, Hemayat-Talab R, Arab-Ameri E, Keyhani F. The effect of physical exercise on the development of gross motor skills in children with attention deficit/hyperactivity disorder. Zahedan J Res Med Sci. 2013;15(2):74-8. [Persian]

19- Rosa Neto F, Goulardins JB, Rigoli D, Piek JP, Oliveira JA. Motor development of children with attention deficit hyperactivity disorder. Rev Bras Psychiatr. 2015;37(3):228-34.

20- Mikami AY, Hinshaw SP, Patterson KA, Lee JC. Eating pathology among adolescent girls with attentiondeficit/hyperactivity disorder. J Abnorm Psychol. 2008;117(1):225-35

21- Cortese S, Renato C, Maia M, Rohde LA, MorcilloPeñalver C, Faraone SV. Prevalence of obesity in attention-deficit/hyperactivity disorder: study protocol for a systematic review and meta-analysis. BMJ Open. 2014;4(3):e00454.

22- Bazar KA, Yun AJ, Lee PY, Daniel SM, Doux JD. Obesity and ADHD may represent different manifestations of a common environmental oversampling syndrome: A model for revealing mechanistic overlap among cognitive, metabolic, and inflammatory disorders. Med Hypotheses. 2006;66(2):263-9.

23- Najafi M, Botshekan R, Mirmahdi R. Investigation of the effect of the using of Kinect-Xbox game console in changing the weight of children with ADHD disorders which use drug Treatment. J Res Behave Sci. 2014;11(5):455-64. [Persian]

24- Davis C. Attention-deficit/hyperactivity disorder: associations with overeating and obesity. Curr Psychiatr Rep. 2010;12(5):389-95.

25- Fragala-Pinkham M, Haley SM, O'Neil ME. Group aquatic aerobic exercise for children with disabilities. Dev Med Child Neurol. 2008;50(11):822-7.

$$
\begin{aligned}
& \text { نتيجهاكيرى } \\
& \text { צ هفته تمرين تناوبى شديد موجب بهبيود عملكرد ادراكى- حركتى }
\end{aligned}
$$

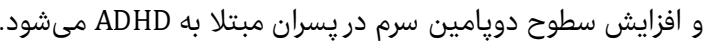

$$
\begin{aligned}
& \text { تشكر و قدردانى: از مسئولان محترم مدارس، معلمان، دانش آموزان }
\end{aligned}
$$

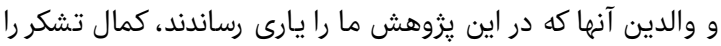

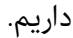

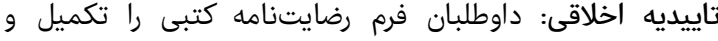

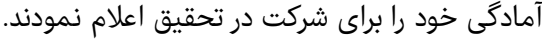

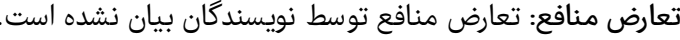

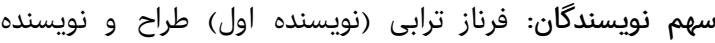

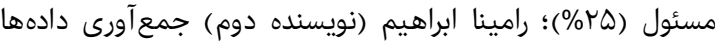

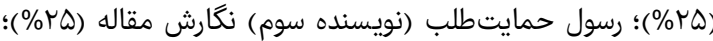

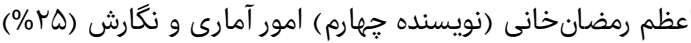

$$
\begin{aligned}
& \text { منابع مالى: مطالعه حاضر بدون حمايت مالى انجام شده است. } \\
& \text { منابع }
\end{aligned}
$$

1- Alizadeh H. Theoretical explanation of the disorder, attention deficit/hyperactivity over pattern of behavioral inhibition and self-control nature. Res Except Child. 2005;5(3):323-43. [Persian]

2- Biederman J, Faraone SV. Attention-deficit hyperactivity disorder. Lancet. 2005;366(9481):237-48. 3- Webster-Stratton CH, Reid MJ, Beauchaine T. Combining parent and child training for young children with ADHD. J Clin Child Adolesc Psychol. 2011;40(2):191-203

4- Harvey WJ, Reid G, Bloom GA, Staples K, Grizenko N, Mbekou V, et al. Physical activity experiences of boys with and without ADHD. Adapt Phys Activ Q. 2009;26(2):131-50.

5- Pan CY, Chang YK, Tsai CL, Chu CH, Cheng YW, Sung MC. Effects of physical activity intervention on motor proficiency and physical fitness in children with ADHD: An exploratory study. J Atten Disord. 2014. pii: 1087054714533192

6- Gupta R, Kar BR. Development of attentional processes in ADHD and normal children. Prog Brain Res. 2009;176:259-76.

7- Mahon AD, Woodruff ME, Horn MP, Marjerrison AD, Cole AS. Effect of stimulant medication use by children with adhd on heart rate and perceived exertion. Adapt Phys Activ Q. 2012;29(2):151-60.

8- Findling RL, Short EJ, Manos MJ. Short term cardiovascular effects of methylphenidate and adderall. J Am Acad Child Adolesc Psychiatry. 2001; 40(5):525-9.

9- Bakhshipour A, Rahnama H, Sourtichi Z, Eskandari S. Influence of the aerobic exercise plan and group theraplay on the balance of children with attention deficit and hyperactivity. J Res Rehabil Sci. 2013;9(2):161-70. [Persian]

10- Ko IG, Kim SE, Kim TW, Ji ES, Shin MS, Kim CJ, et al. Swimming exercise alleviates the symptoms of attentiondeficit hyperactivity disorder in spontaneous hypertensive rats. Mol Med Rep. 2013;8(2):393-400.

11- Johnson MH, Gliga T, Jones E Charman T. Annual 